\title{
Gastric Pouch After Simple or Radical Cystectomy for Benign and Malignant Bladder Disease
}

\author{
Ali Shamsa ${ }^{1, *}$ \\ ${ }^{1}$ Mashhad University of Medical Sciences, Mashhad, IR Iran \\ ${ }^{*}$ Corresponding author: Ali Shamsa, Mashhad University of Medical Sciences, Mashhad, IR Iran. Tel: 98-511802857, E-mail: shamsaa@mums.ac.ir \\ Received: January 30, 2014; Revised: July 27, 2014; Accepted: August 11, 2014
}

\begin{abstract}
Background: There is a controversy regarding the use of gastric pouch for benign and malignant bladder diseases.
Objectives: The aim of this retrospective study was to report our experience, mostly on gastric neobladder (not gastrocystoplasty) for benign and malignant bladder diseases, its complications, outcomes and follow-up results.

Materials and Methods: In this retrospective case series, we described our experience on seven gastric pouches (2 gastrocystoplasty and 5 gastric neobladders).

Results: Postoperative complications were rare. Continence was defined as bladder capacity over $400 \mathrm{~mL}$. Their follow-up period ranged from five months up to writing the article. One of the studied cases is still alive and awaiting renal transplantation.

Conclusions: Gastric pouch is a suitable segment for bladder cystoplasty and neobladder. Continence is mostly related to the capacity of pouch.
\end{abstract}

Keywords:Stomach; Gastric Pouch; Gastroblasty

\section{Background}

A variety of techniques have been introduced for making orthotopic neobladder after simple and radical cystectomy for benign, invasive or recurrent bladder tumors, such as Ileum, colon and sigmoid. Nonetheless, a controversy exists and there are scarce reports in the literature on the use of gastric pouch for this purpose. Some are against it (1), some advocate its application for certain and specific patients (2), and some others believe that the use of bowel has some limitations including excessive mucus production, metabolic acidosis, stone formation, infection, perforation and malignancy (3). There are some advantages for using a pouch of stomach for cystoplasty and gastric neobladder for example:

1- Reimplantation of ureter to this segment is easer, because of its thickness.

2- Because stomach is beyond the field of radiotherapy and free of adhesion.

3- Stomach is an organ of choice for diversion during chronic renal failure (CRF).

In the current study we used gastric pouch for augmentation cystoplasty in transitional cell carcinoma (TCC) of bladder for the first time in Khorasan province (4), also benign diseases of bladder like severe contracted bladder due to tuberculosis and failed vesicovaginal fistula (5). In addition, cases of gastric neobladder for malignant disease for malignant of bladder were studied. Patients with incomplete records were excluded from this study.

\section{Objectives}

The aim of this retrospective study was to report our experience, mostly on gastric neobladder (not gastrocystoplasty) for benign and malignant bladder diseases, its complications, outcomes and follow-up results.

\section{Materials and Methods}

From Oct.1992 to Aug.1996, seven cases of gastric pouch were performed in the Urology Department of Qaem Educational Hospital, Mashhad, Iran. An informed consent was obtained from each case prior to study enrollment.

Two of the seven cases were male with severe tuberculosis cystitis. Their age ranged from 40 to 72 years. Five cases of invasive bladder tumor, aged between 40-70 (mean: 61.6) years were selected for radical cystectomy after performing 1-3 times transuretheral resection of bladder tumor (TURBT). All patients underwent staging before cystectomy including TURBT, histopathologic studies, bimanual exam under anesthesia, abdominal CT, chest X Ray and renal and liver tests. Patient No. 1, A.A., a 62-year-old male; TCC of bladder, stage B2, grade 2. He had a huge tumor on the bladder dome. Patient No. 2, M.B., a 62-year-old female; TCC of bladder, stage B2, grade 3. Patient No. 3, M.M.K., a 51-year-old male; recurrent multiple papillary tumor, involving trigone and lateral walls, stage A, grade 2 (history of repeated TURBT in a short period of time). Patient No. 4, G.A., a 
70-year-old male with TCC of bladder, stage B2, Grade 2. Patient No. 5, A.A.K., a 63-year-old male with TCC of bladder, stage B2, Grade2. All patients underwent cystourethroscopy (including evaluation of urethra) and biopsy from suspicious areas was performed; however, no in situ carcinoma was diagnosed in any case. Patients No. 6 and 7 had urinary tuberculosis (TB) with positive results for urine culture.

Patient No. 6 was a 72-year-old man who had irritative symptoms plus gross painless hematuria and nearly true urinary incontinence. His IVP (intra venous pyelography) showed ureterovesical junction (UVJ) stenosis with a severely contracted (thimble) bladder. After eight weeks of anti-TB therapy because of a $1.7 \mathrm{mg} / 100$ creatinine level (normal: 0.5-1.5 mg/100), he underwent gastric neobladder.

Patient No. 7 was a 40-year-old man from Afghanistan with a right hydronephrotic kidney with $5 \%$ function on DMSA and grade three reflux on the left side in voiding cystourethrography (VCUG).

Because of left hydroureteronephrosis, percutaneous nephrostomy (PCN) was performed on the left side. Creatinine decreased from $5.1 \mathrm{mg} / 100 \mathrm{~mL}$ to $4.2 \mathrm{mg} / 100 \mathrm{~mL}$ and nephrostography showed a left-sided stenosis. He had nearly true urinary incontinence with a low bladder capacity. For this case gastrocystoplasty and left ureteral reimplantation were performed.

Preoperative regimen was started for all patients, three days prior to the operation. It consisted of liquid food as well as bisacodyl, 2 tablets a day, enema at night with normal saline, plus metronidazol 2 tablets every 8 hours. Patients were NPO ("nil per os" meaning nothing through mouth) from 12 hours before the procedure and received broad spectrum antibiotics intraparentally.

\subsection{The Procedure Technique}

Laparotomy was performed with a midline incision from xyphoid through pubis. Routine exploration of intra-abdominal organs was performed as well as bladder mobility evaluations.

Radical cystectomy was performed for patients No. 2, 3, 4 and 5. Regional lymphadenectomy was performed for patients No. 2 and 3, but for patients No. 4 and 5, frozen section of a suspicious lymph node had negative results, therefore lymphadenectomy was not performed. Histopathology study of regional lymph nodes of patients No. 2 and 3 had negative results as well.

In patients No. 3, 4 and 5 who were male, total bladder, urachus, seminal vesicles, prostatic urethra, up to the prostatic apex were removed leaving a stump from the membranous urethra. No attempt was made to preserve the cavernousal nerve in male patients. All ureters were cut from $2 \mathrm{~cm}$ from the bladder, except for patient No. 1, in whom only partial cystectomy with $2 \mathrm{~cm}$ free margin was performed.

For patient No. 2 (female case), anterior pelvic exentera- tion with hysterectomy, oopherectomy and salpingectomy were performed leaving only a partial section of vagina and urethral stump.

Gastric wedge was performed according to the Michell technique (6). Simple cystectomy was performed for patient No. 6 and gastrocystoplasty for patient No. 7 whom had a thimble bladder.

By marking a triangle on the fundus of the stomach with methylene blue, a $15 \mathrm{~cm}$ long base (in our first experience) and then $20 \mathrm{~cm}$ long (after the first case) far from antrum to the fundus was selected. Its vasculature for all cases was based on the right gasteroepiploic artery. Stomach was closed in two layers. Gastric wedge was brought to the pelvis by passing it through the transverse mesocolon and small bowel mesentery in a straight direction (Figures 1,2).

\subsection{Case by Technique}

In case No.1, there was no need for ureteral anastomosis.

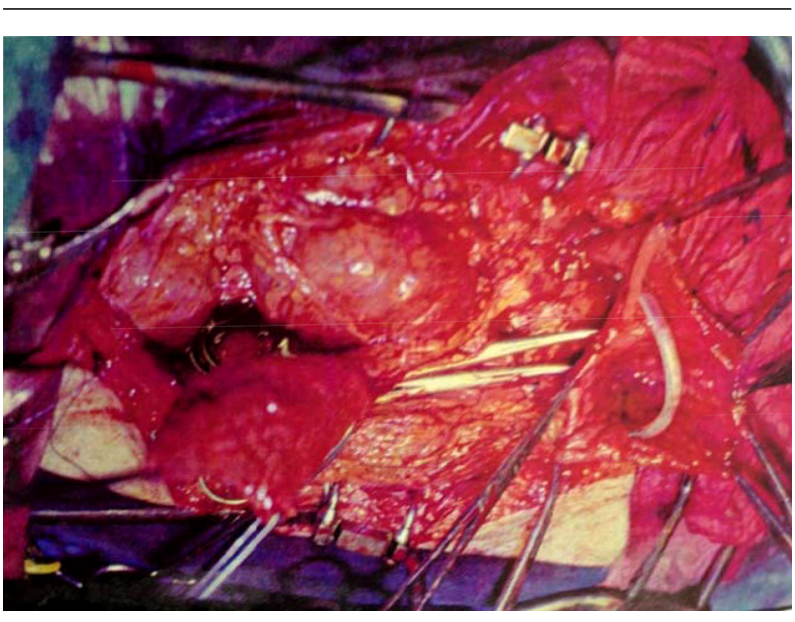

Figure 1. Gastric Flap Is Excised. Nelaton catheter is in the stomach.

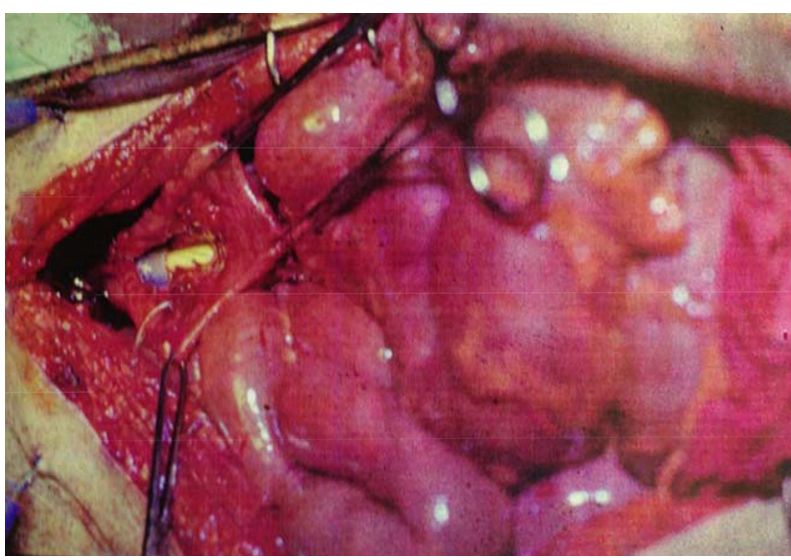

Figure 2. Gastric Pouch, Anastomosed to Urethra. Foley catheter is visible 
In this case the gastric wedge was anastomosed to the bivalve bladder, after partial cystectomy. In patients No. 2 and 3, the gastric pouch was anastomosed to the urethra, and then the two ureters in each case were anastomosed to the new bladder. Two 8Fr feeding tubes were put into the ureters as a stent and a three way 22Fr Foley catheter inside the bladder (a D.J. catheter was not available at that time). A cystostomy tube was neither used nor advocated. In patients No. 4 and 5, because of a small flap and a short pedicle, a distal part of the flap was tubularized around a 22 three way catheter and was anastomosed to the membraneous urethra by six sutures with 2-0 chromic catgut. At the end of the operation the bladder was filled with saline solution to evaluate neobladder volume and also urinary leakage. Hemovac drain was inserted for perivesical drainage. The operation time for patients 1 to 7 were 3, 4.52, 5.5, 6, 5, 6 and 10 hours, respectively (mean: 325 minutes).

Intravenous cimetidine $200 \mathrm{mg}$ four times a day, was administered after the operation for 4-5 weeks. Antimicrobial therapy way performed to two weeks following catheter removal. NG (Nasogastric) tubes were removed after gas passing. Fluid regimen was started one day after NG removal.

Ureteral catheter was removed one week after the operation, and Hemovac drain one day later (if the amount of secretion was less than $30 \mathrm{~mL}$ ). Bladder irrigation was performed continuously for the first and second patients, but for patients 3 to 7, it was stopped. However, after getting more experience in benign conditions (T.B.), bladder irrigation was stopped. We never used a cystectomy tube. Foley catheter was removed four weeks after the operation, after retrograde cystography to assess leakage.

All patients started walking from the third post-op day except patient No. 5, who experienced a longer operation time and post-op tachyarrhythmia. He was advised to have complete bed rest until five days post-op.

\section{Results}

Fortunately, rare complications occurred from such massive and pr operations. Expected complications were summarized in Table 1 . All patients were followed up by renal and bladder US (Ultrasonography), IVP (intravenous pyelography) and metabolic assay by measuring $\mathrm{Na}, \mathrm{K}$, bicarbonate, blood and urine $\mathrm{pH}$, and in one case measuring serum Gastrin. All the studied cases had normal renal function and electrolyte levels.

\subsection{Follow-up}

Patients No. 1, 2, 5, 6 and 7 were living in the current city and had a more precise follow-up compared to patients No. 2 and 4 who lived in other cities. Evaluation of the pouch for infection was performed by periodic urine culture. Three of the 25 urine cultures had positive results (12\% Bacteriuria). Our follow-up period was 5-36 (mean: 20.2) months. In these cases we had one dysuria hematuria syndrome (which is more common in pediatric patients), who had undergone gastrocystoplasty after failed vesicovaginal and contracted bladder. In this case, we prescribed $\mathrm{H} 2$ blocker, continuously.

\subsection{Urodynamic Study}

As we had no urodynamic facility at that time, only one case was sent to a higher advanced center for this purpose and the results are presented, but their bladders capacity and residual urine were measured. Its results are as follows: the neobladder volume was $400 \mathrm{~mL}$ in patients 1, 2, 4 and 5 but in cases 3, 6 and 7, were 310,100 and $350 \mathrm{~mL}$, respectively. Final follow-up for patients No. 1, 3 and 5 were 36,24 and 5 months, respectively, and all were in a good condition until that time. In patient No. 2, SCC appeared 27 months post-op. He rejected operation and died two months later. Patient No. 4 also died of latent metastasis 7 months post-op.

Table 1. Post-Operative Complications

\begin{tabular}{|c|c|c|c|c|c|c|c|}
\hline \multirow[t]{2}{*}{ Complication } & \multicolumn{7}{|c|}{ Patient No. } \\
\hline & $\mathbf{1}$ & 2 & 3 & 4 & 5 & 6 & 7 \\
\hline Mortality & - & - & - & - & - & - & - \\
\hline Re-operation & - & - & - & - & - & - & - \\
\hline Abscess formation and peritonitis & - & - & - & - & - & - & - \\
\hline Respiratory complications & - & - & - & - & - & - & - \\
\hline Dehiscence & - & - & - & - & - & - & - \\
\hline Hemorrhage & + & - & - & - & - & - & + \\
\hline Fluid collection & - & - & - & - & - & - & - \\
\hline Fistula & - & - & + & - & - & - & - \\
\hline Food intolerance & - & - & - & - & - & - & - \\
\hline Blood transfusion ${ }^{a}$ & + & + & + & + & +++ & + & +++ \\
\hline
\end{tabular}

a +++ Referred to More Than Average Blood Transfusion. 


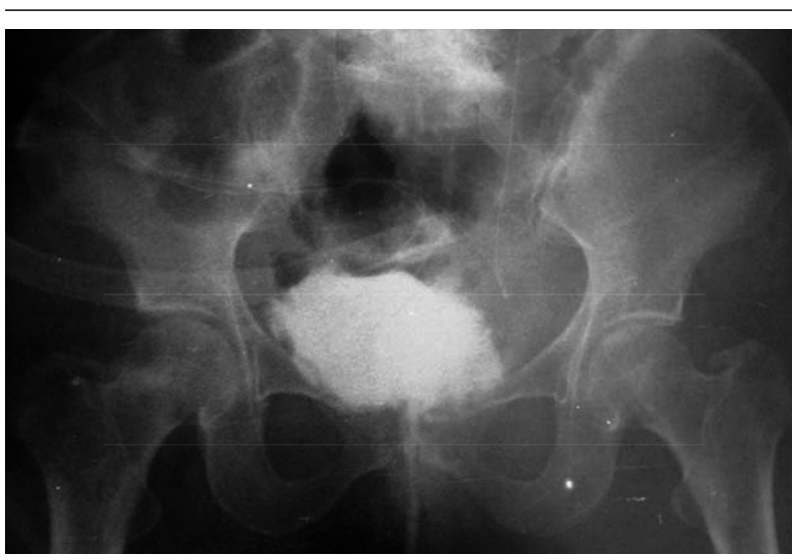

Figure 3. Cryptogram of Patient No. 2 Two Months After Gastric Neobladder

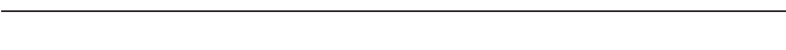

Figure 4. IVP of Patient No. 3 One Year Post-Gastric Neobladder

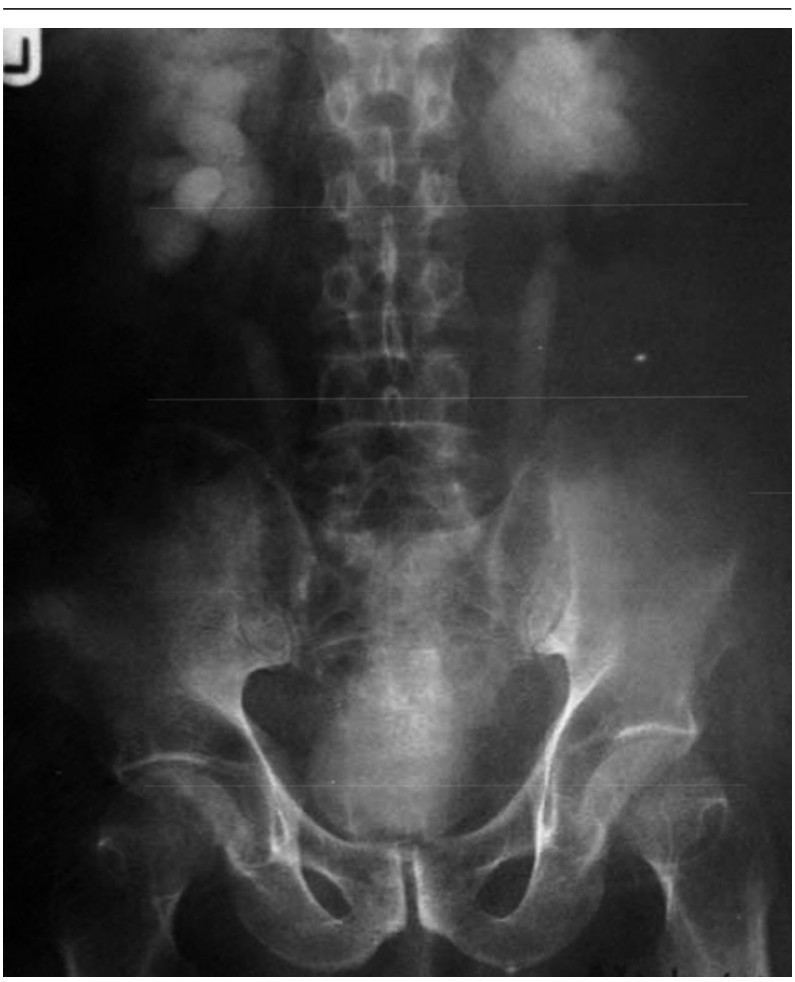

Bilateral hydroureteronephrosis; probable ureterogastric stenosis.

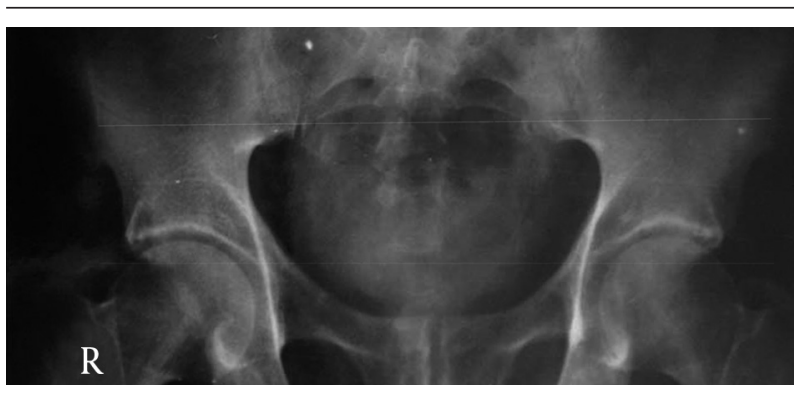

Figure 5. Cystogram, Patient No. 3, Bladder Volume Is Very Good

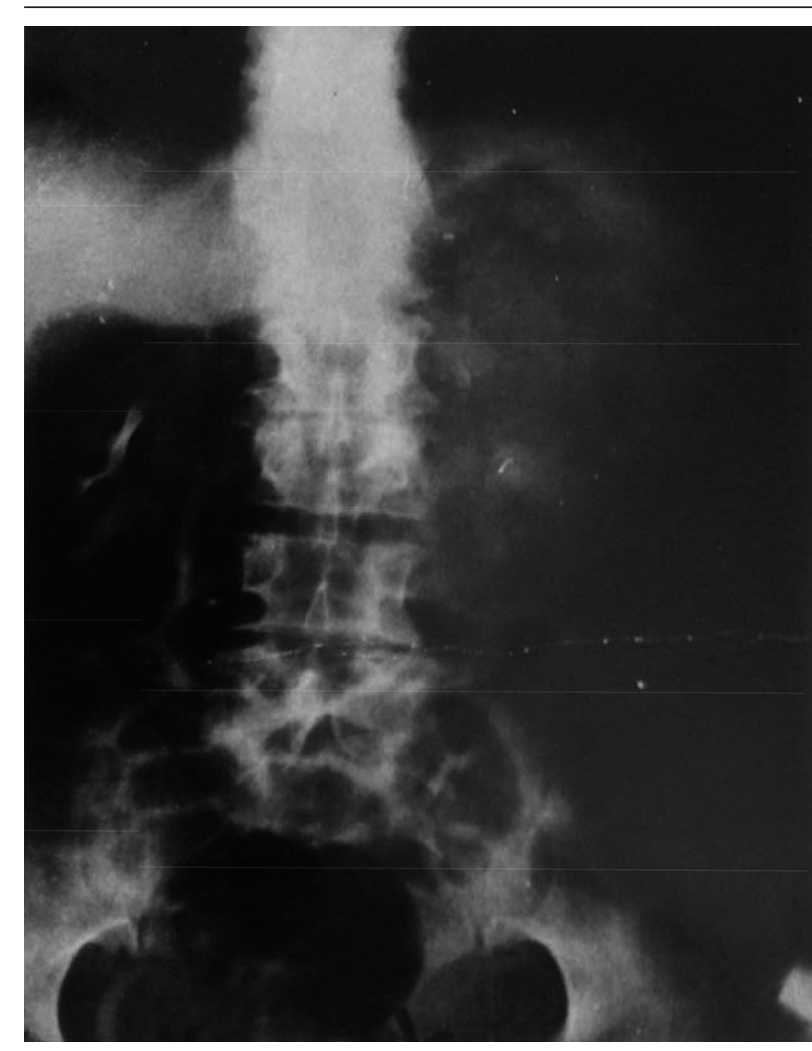

Figure 6. IVP Patient No. 4, Six Months Postoperation. Right renal function was normal. Left kidney was non-functioning due to tuberculosis.

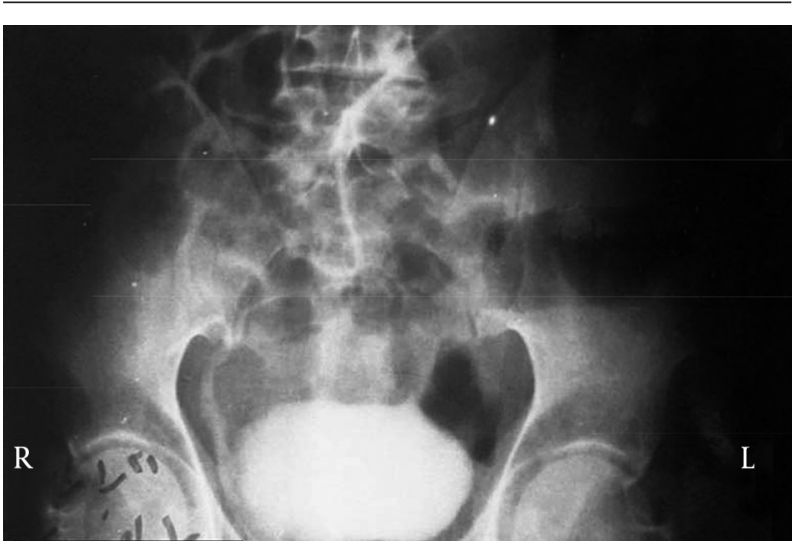

Figure 7. Cystogram patient No. 4 Two Months Post-Gastric Neobladder

Patient No. 6 died two years post operatively because of a heart attack. Patient No. 7 was alive with end stage renal disease and awaiting renal transplantation up to writing the article (we have no waiting list for Iranian patients but as a law, a foreigner must introduce a relative live donor from his or her country). One of our patients was a woman who received a urethral catheter due to urinary urge incontinence. The rest of our patients had acceptable levels of urinary continence (Figures 3 -12). 


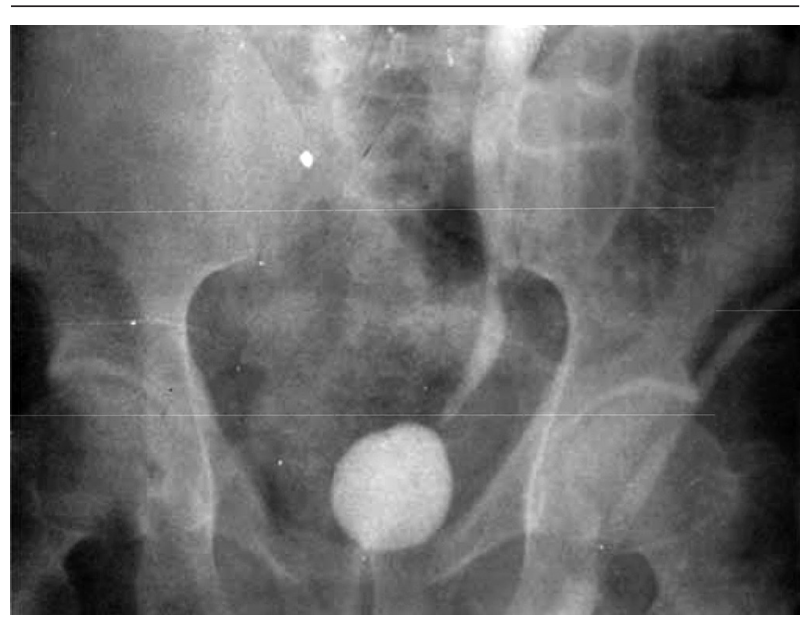

Figure 8. Retrograde Cystography Patient No. 5 One Year Post-Gastrocystoplasty Ureteovesical Reflux Disappeared

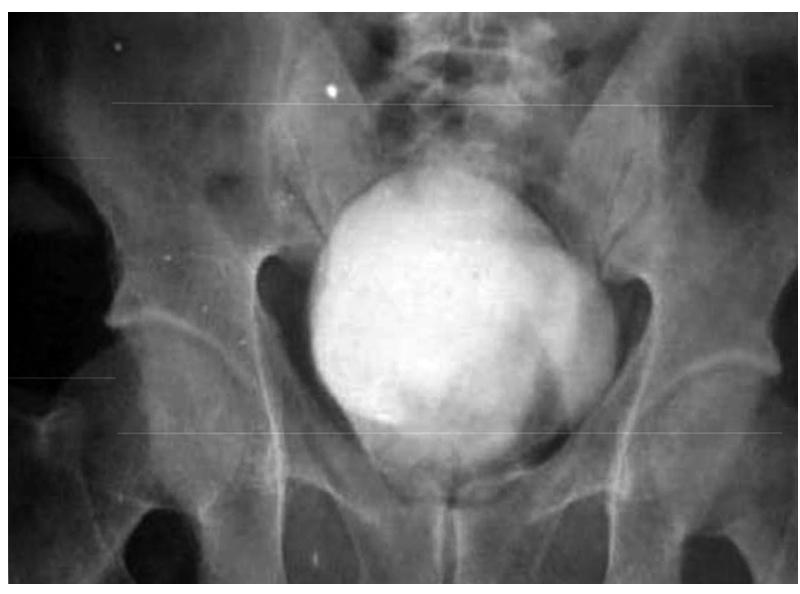

Figure 9. Retrograde Cystography Patient No. 5 Before the Operation, Left Vesicoureteral Reflux Is Visible

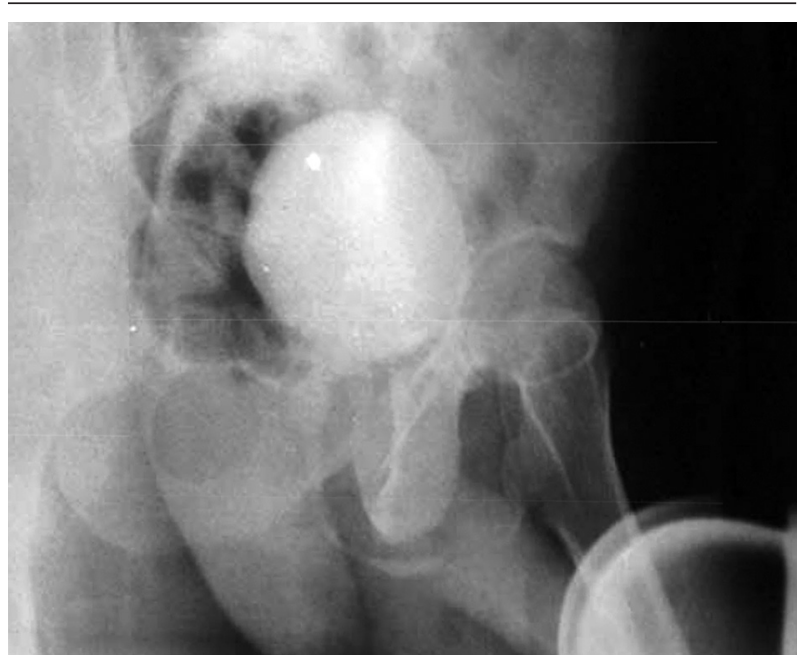

Figure 10. Voiding Cystouretrography, Patient No. 5 One Year Postoperation Can Void and Whole Urethra Is Visible

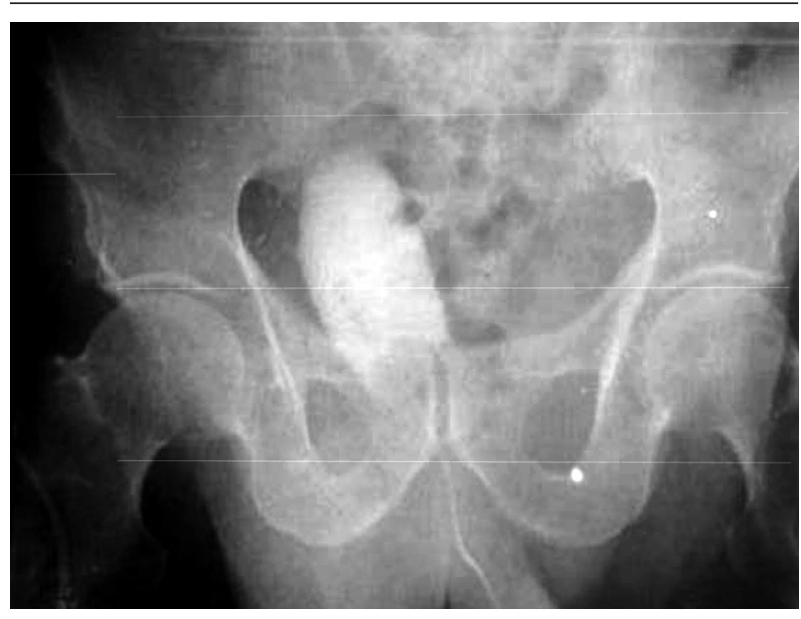

Figure 11. Retrograde cystography patient No. 6, Two Months Post-Gastric Neobladder. Neobladder volume is less.

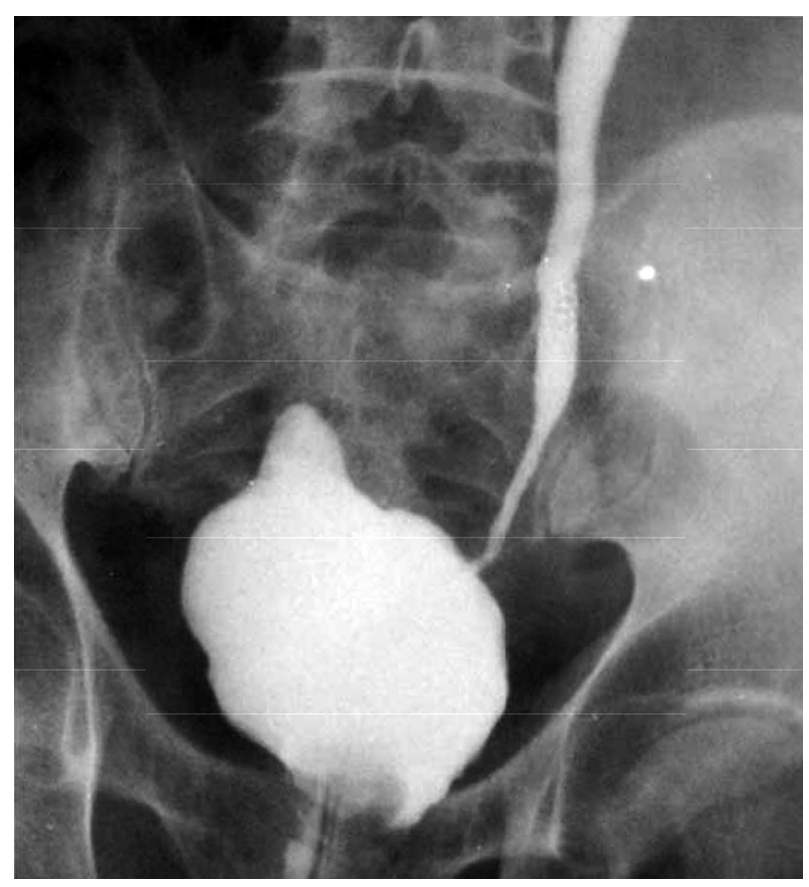

Figure 12. Retrograde Cystography Patient No. 7 Two Months Post-Gastric Neobladder. Neobladder volume is good.

\section{Discussion}

Sinaiko performed the first gastric neobladder in 1956 after partial cystectomy in a patient with bladder tumor (7); then Leong used a gastric pouch for augmentation after tuberculosis cystitis and bladder tumor (8). Since then a few articles were published on advantages and disadvantages of this type of procedure. All authors believe that it is a good segment for bladder replacement in boys (9), as well as adults after cystectomy for benign (5) and malignant diseases (10). However, it is still controversial weather to use it as a neobladder in adults after radical 
cystectomy for bladder tumors. For example Lin et al. believed that adult gastric neobladder is associated with poor urodynamic parameters and high incontinence rates (1). On the other hand, Koraitim et al. reported that stomach is an ideal and suitable segment for bladder augmentation and/or creating a neobladder (10).

Compared to other segments of the gastrointestinal tract, stomach has a thicker wall, which makes it easy to perform antireflux ureteral anastomosis. Furthermore, in patients with invasive bladder tumor who receive radiotherapy, stomach is far from the field of radiotherapy compared to other parts of the gastrointestinal tract. Moreover, it makes less mucus production, so suprapubic catheter in gastric neobladder is not necessary (5); and the last but not least, stomach is impermeable to most ions especially chloride, which is transported in urine (10); thus, it has a protective effect against acidosis in patients with renal impairment. One of the most common complications of gastric neobladder or gastrocystoplasty is perforation of the pouch. Studies showed that perforation in gastric pouch is negligible compared to Ileal, ileocaecal or sigmoid ones. Therefore, it is an ideal segment in patients with concomitant renal failure and invasive bladder tumors. Our mean operative time was $325 \mathrm{~min}$ utes (180-600) compared to 365 (300-450) minutes in Wang et al. study with more than 20 years of experience on gastric replacement therapy (3).

There are many intraoperative and postoperative complications such as blood loss, hematemesis, intestinal adhesion, increased serum gastric and cardiac failure (one case, due to anesthetic problem, is not in this series).

Santucci et al. performed seven gastric neobladders and concluded that gastric pouch has a smaller capacity with greater incontinence rates (2). They performed urodynamic study after an average of 9.1 months and reported the volume of neobladder as $311 \mathrm{~mL}$.

Lin et al. performed eight gastric neobladders (malignancy in seven and undiversion in one) with an average follow-up of 9.1 months. They reported that gastric neobladder capacity reduced to a mean of $309 \mathrm{~mL}$ and concluded that routine use of gastric neobladder in adults is not recommended (1).

Capacity of neobladder is an important factor for incontinence and involuntary contraction (11). The capacity of gastric neobladder like other neobladders might increase postoperatively (11). The final capacity of gastric neobladder can be determined six months (11) and one and a half years after the operation (12). Wang et al. reported their experiences on nine cases of gastric neobladder by per- forming it after laparoscopic radical cystectomy, showing a good capacity of pouch and continence (3).

Gastric neobladder is an ideal procedure after cystectomy for bladder benign and malignant diseases. To prevent ureteral stenosis, we should have adopted to do tension free ureteral reimplant; however, it is more challenging compared to the rate of continence and is totally related to volume of neobladder and surface of the wedge. When the volume and surface of the gastric wedge is high, the rate of continence is greater. Nevertheless, we recommend investigating more patients with longer and better follow-up including urodynamic study and gastrin measurement.

\section{Acknowledgements}

I would acknowledge Omid hospital and its operation theater team.

\section{References}

1. Lin DW, Santucci RA, Mayo ME, Lange PH, Mitchell ME. Urodynamic evaluation and long-term results of the orthotopic gastric neobladder in men.J Urol. 2000;164(2):356-9.

2. Santucci RA, Park CH, Mayo ME, Lange PH. Continence and urodynamic parameters of continent urinary reservoirs: comparison of gastric, ileal, ileocolic, right colon, and sigmoid segments. Urology. 1999;54(2):252-7.

3. Wang XH, Pu XY, Wang HP, Hu LQ. Laparoscopic radical cystectomy with orthotopic gastric neobladder: technique and initial outcomes. J Cancer Res Clin Oncol. 2009;135(2):197-202.

4. Shamsa A. Video presentation: gastric neobladder. British JUrolog .1997;80(2):354.

5. Shamsa A. Gastric neobladder for treatment of tuberculosis cystitis. JUrol.1998;159(1):202.

6. Adams MC, Mitchell ME, Rink RC. Gastrocystoplasty: an alternative solution to the problem of urological reconstruction in the severely compromised patient. J Urol. 1988;140(5 Pt 2):1152-6.

7. Sinaiko E. Artificial bladder from segment of stomach and study of effect of urine on gastric secretion. Surg Gynecol obstet. 1956;102(4):433-8.

8. Leong $\mathrm{CH}, \mathrm{YU} \mathrm{H}$. A Comparison between pre and postoperative pelvic irradiation using curative dosage and radical surgery alone for the treatment of advanced cancer of the urinary bladder. Bull Soc Int Chir::6/506-11.

9. DeFoor WR, Heshmat S, Minevich E, Reddy P, Koyle M, Sheldon C. Long-term outcomes of the neobladder in pediatric continent urinary reconstruction. J Urol. 2009;181(6):2689-93.

10. Koraitim MM, Khalil MR, Ali GA, Foda MK. Micturition after gastrocystoplasty and gastric bladder replacement. J Urol. 1999;161(5):1480-4.

11. Keszthelyi A, Majoros A, Nyirady P, Mayer P, Bach D, Romics I Voiding symptoms and urodynamic findings in patients with modified ileal neobladde. Pathol Oncol Res. 2009;15(3):307-13.

12. Shamsa A, Tavassoli M. Gastorocystoplasty, report of a first case in Khorasan Province. Med. J. Mashad Uni of Medic Sci. 1994;37(46):103-5. 